ARTICLE

Received 31 May 2016 | Accepted 21 Jul 2016 | Published 31 Aug 2016

DOI: $10.1038 /$ ncomms12672

OPEN

\title{
Magnetic measurements with atomic-plane resolution
}

Ján Rusz' ${ }^{1}$ Shunsuke Muto², Jakob Spiegelberg${ }^{1}$, Roman Adam $^{3}$, Kazuyoshi Tatsumi², Daniel E. Bürgler ${ }^{3}$, Peter M. Oppeneer ${ }^{1} \&$ Claus M. Schneider ${ }^{3}$

Rapid development of magnetic nanotechnologies calls for experimental techniques capable of providing magnetic information with subnanometre spatial resolution. Available probes of magnetism either detect only surface properties, such as spin-polarized scanning tunnelling microscopy, magnetic force microscopy or spin-polarized low-energy electron microscopy, or they are bulk probes with limited spatial resolution or quantitativeness, such as X-ray magnetic circular dichroism or classical electron magnetic circular dichroism (EMCD). Atomic resolution EMCD methods have been proposed, although not yet experimentally realized. Here, we demonstrate an EMCD technique with an atomic size electron probe utilizing a probe-corrected scanning transmission electron microscope in its standard operation mode. The crucial element of the method is a ramp in the phase of the electron beam wavefunction, introduced by a controlled beam displacement. We detect EMCD signals with atomic-plane resolution, thereby bringing near-atomic resolution magnetic circular dichroism spectroscopy to hundreds of laboratories worldwide.

\footnotetext{
${ }^{1}$ Department of Physics and Astronomy, Uppsala University, Box 516, S-75120 Uppsala, Sweden. ${ }^{2}$ Advanced Measurement Technology Center, Institute of Materials and Systems for Sustainability, Nagoya University, Chikusa-ku, Nagoya 464-8603, Japan. ${ }^{3}$ Peter Grünberg Institute, Electronic Properties (PGI-6), Forschungszentrum Jülich, 52425 Jülich, Germany. Correspondence and requests for materials should be addressed to J.R. (email: jan.rusz@physics.uu.se) or to S.M. (email: smuto@imass.nagoya-u.ac.jp).
} 
$\mathrm{M}$ agnetic circular dichroism in electron microscopy, electron magnetic circular dichroism (EMCD), was first observed in 2006 (ref. 1), following swiftly its prediction from 2003 (ref. 2). The technique attracted a lot of interest due to its analogy to X-ray magnetic circular dichroism ${ }^{3}$, as well as for its potential of reaching atomic resolution routinely available in probe-corrected scanning transmission electron microscopy (STEM). Since the inception of EMCD, a rapid development has followed, deepening the theoretical understanding ${ }^{4-6}$, initiating development of new experimental set-ups and improving the spatial resolution and quantitativeness $^{7-14}$. However, the goal of achieving atomic resolution remains unaccomplished. The reasons are practical limitations of the classical EMCD technique combined with the low signal-to-noise ratios. In classical EMCD (ref. 1), the sample is tilted to a 2-beam or 3-beam orientation and signal acquisition is done on Thales circle positions aside the Bragg spots, greatly wasting electron counts. The resulting low-count rates then combine unfavourably with limited spectrum acquisition times in atomic resolution STEM, which are required to prevent sample damage or drifts. New inspiration came in 2010 when the generation of electron vortex beams was demonstrated ${ }^{15-17}$. As shown later by theory ${ }^{18,19}$, it should be possible to detect EMCD at atomic resolution with electron vortex beams having a non-zero orbital angular momentum. Notably, for this approach the atomic size of the probe is a requirement, not an option. Theoretical analysis has shown that the symmetry of the phase distribution in the wavefunction of the electron beam plays a crucial role ${ }^{20}$ in detection of EMCD. As a consequence, alternative beam shapes have been proposed for atomic resolution EMCD, such as astigmatic beams ${ }^{20}$. Yet, even today an EMCD detection with atomic size probes remains very demanding. The likely reasons are technical difficulties in generating atomic size electron vortex beams, together with fine control of their resulting orbital angular momentum in the case of electron vortex beams, and a low EMCD signal fraction in the case of astigmatic beams ${ }^{22}$.

In this work we demonstrate measurements of EMCD with atomic-plane resolution (APR-EMCD). Our approach combines ideas of both classical and atomic resolution EMCD. Theoretical formulation is supported by explicit simulations and experiments. The technique does not require any modifications of the microscope hardware and is thus accessible to hundreds of laboratories worldwide.

\section{Results}

Theory. The APR-EMCD combines ideas of both classical and atomic resolution EMCD. From classical EMCD we borrow the concept of tilting the sample into a 3-beam orientation, see Fig. 1a. The advantage is a simplification of the dynamical diffraction effects, compared with zone-axis orientation. The price paid is that we lose the view of individual atomic columns, instead we can observe atomic planes as parallel lines in a high-angle annular dark field (ADF) STEM image, Fig. 1b,c. From the atomic resolution EMCD approaches we borrow the concept of manipulating the phase distribution in the probe wavefunction. Naturally this requires to use large convergence angles, leading to a coherent overlap of the discs of the diffracted beams. Following ref. 20, for a magnetization $\mathbf{M}=(0,0, M)$ parallel to the beam direction and Bragg spots $\mathbf{G} \pm= \pm(G, 0,0)$ along the $x$ axis, the EMCD signals originate primarily from mixed dynamical formfactors 22 proportional to the $y$-component of the momentum transfer $q_{\mathrm{y}}$ (see Methods section for a detailed derivation). These terms are multiplied by the sines of the phase difference $\Delta \phi_{\mathbf{k}, \mathbf{G}}=\phi_{\mathbf{k}+\mathbf{G}}-\phi_{\mathbf{k}}$. A plain shifting of the beam introduces a phase ramp $e^{2 \pi i\left(k_{x} \Delta x+k_{y} \Delta y\right)}$ in the Fourier transformed wavefunction, as a trivial consequence of the Fourier shift theorem. Considering that the Bragg spots lie along the $x$ axis in our reference frame, only the shift in $x$-direction matters, resulting in a phase difference of $\Delta \phi_{\mathbf{k}, \mathbf{G}}=2 \pi G \Delta x$ independent of $k_{x}, k_{y}$. The sine of such phase differences is maximized/ minimzed when the beam shift $\Delta x$ equals $\pm \frac{d}{4}$, where $d$ is the atomic-plane spacing. In other words, in the 3-beam orientation we expect to detect positive and negative EMCD signals in the neighbourhood of the atomic planes. The proportionality of the magnetic signal to $q_{\mathrm{y}}$ means that the detection can be done in the upper or lower diffraction half-plane. A scheme of the experimental set-up and the comparison to a classical EMCD experiment in 3-beam orientation is shown in Fig. 1d.

Let us discuss briefly the importance of this claim. It is proposed that a standard spectrum image measured on a sample tilted into a 3-beam orientation will contain EMCD signals next to the atomic planes. Hence, there is no need of beam shaping with angular momentum or aberrations, because the desired phase differences arise naturally as a consequence of shifting the beam during the scan. In addition, the method provides areas of both positive and negative EMCD in one and the same data set, acquired under identical measurement conditions, eliminating the need to scan the same area twice-once with positive and once with negative angular momentum or astigmatism. Consequently, the proposed APR-EMCD method sidesteps the technical issues associated with atomic resolution EMCD.

Simulations. First we probe the validity of the APR-EMCD method by an explicit simulation of dynamical diffraction effects and inelastic scattering of an atomic size probe $e^{23}$ on an iron crystal tilted to a 3-beam orientation with $\mathbf{G}=(110)$ and the beam parallel to the (1118) direction, which represents a tilt of $10^{\circ}$ from the (001) crystal orientation. Figure 2 summarizes the results. An example distribution of the EMCD signal in the diffraction pattern for a beam shifted to the side of an atomic plane is shown in Fig. 2a. Note how this distribution differs qualitatively from the classical 3-beam EMCD, which displays EMCD of alternating signs in the four quadrants. See Supplementary Fig. 1 for an example of such a diffraction pattern. The rectangular detector was positioned as shown in Fig. $2 \mathrm{a}$ and a wide range of sample thicknesses and convergence angles has been evaluated. The resulting relative EMCD strength is shown in Fig. 2b, from which we conclude that an optimal convergence semi-angle for 20 and $30 \mathrm{~nm}$-thick samples is 15 and $10 \mathrm{mrad}$, respectively. The latter conditions were then used to simulate the thickness and probe-position dependence of the non-magnetic signal, shown in Fig. $2 c$, and the corresponding relative strength of APR-EMCD signal, Fig. 2d, confirming the localization of the APR-EMCD signal at positions $\pm \frac{d}{4}$ distant from the lattice planes.

Experiments. Having theoretically optimized measurement conditions we proceed to the experimental verification. Two samples were examined, a 20 and $30 \mathrm{~nm}$-thick polycrystalline layer of bcc iron with lateral sizes of the grains of the order of $100 \mathrm{~nm}$. A grain oriented in the desired 3-beam condition with $\mathbf{G}=(110)$ has been found and several spectrum images have been acquired. For the further analysis of the data it is advantageous to acquire data in rectangular regions with the short side parallel to the atomic planes and the long side perpendicular to them, see Fig. 1d. Scanning in the direction parallel to the atomic planes one can minimize the effects of sample drift, since only a few pixels (typically $<10$ ) are acquired per scan row. A representative ADF image is shown in Fig. 3 a. 


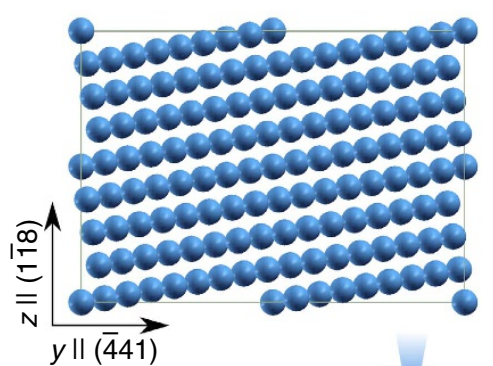

b

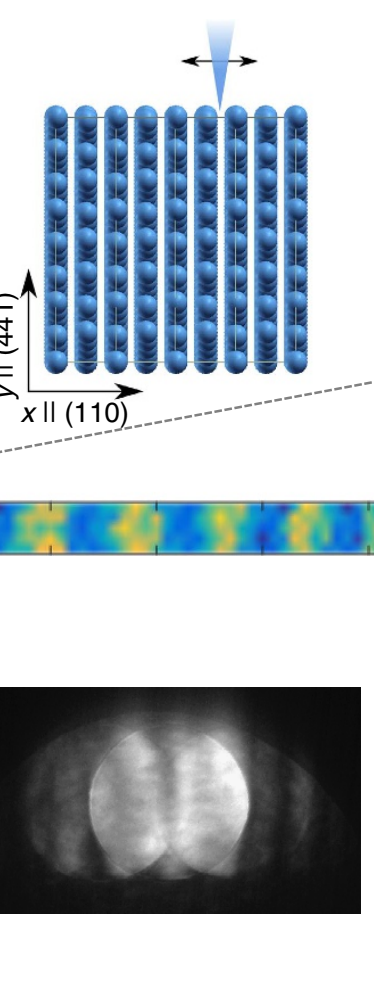

c

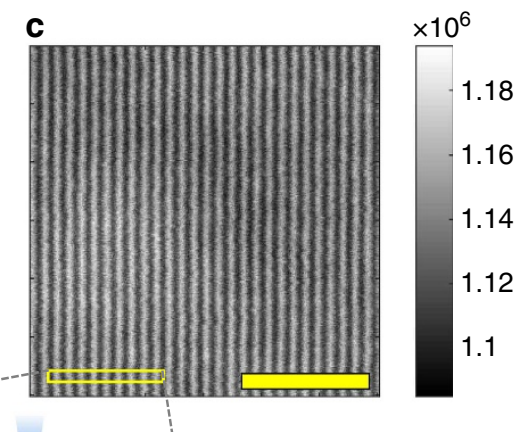

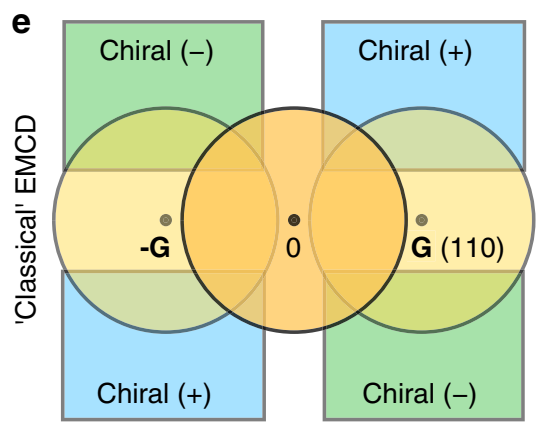

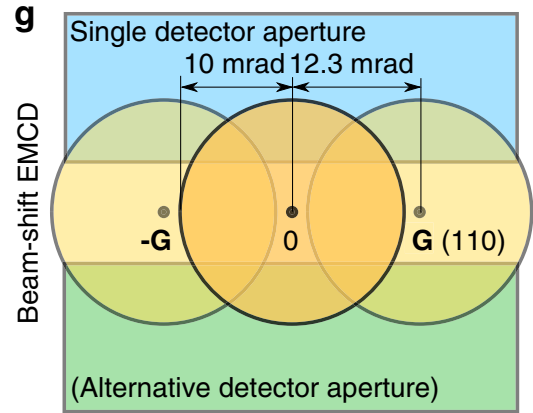

Figure 1 | Scheme of the experimental set-up. (a) Side-view of the structure model of bcc iron tilted into a 3-beam orientation with the beam along the (118) direction. (b) Top view of the structure model. Scanning direction is schematically shown by blue cone with arrows. (c) ADF survey image (acceleration voltage $200 \mathrm{kV}$, convergence semi-angle $10 \mathrm{mrad}$ ) showing atomic planes. The yellow scale bar denotes $2 \AA$. (d) ADF image acquired along with the acquisition of a spectrum image. Its size corresponds to the yellow rectangle marked in the ADF survey image. (e) Schematic placement of detectors for a classical EMCD approach in the 3-beam orientation. (f) Experimental diffraction pattern acquired in the same settings as the ADF survey image. (g) Detector placement in the here-proposed APR-EMCD approach. Note that in the classical approach we need two measurements at each beam position to acquire both chiral $(+)$ and chiral $(-)$ spectra, while in the APR-EMCD a single detector aperture is sufficient.

Data analysis. Due to a very low signal-to-noise ratio in individual spectra, a careful data analysis approach is needed. Details of the noise-reduction procedure are described in the Methods section. Two regions of interest (ROI, Fig. 3c) with a beam shift of $\pm \frac{d}{4}$, respectively, were determined on the basis of the ADF image. Summing all spectra within the region with expected positive/ negative EMCD signals results in two spectra whose difference is expected to show an EMCD signal, as shown in Fig. 3g. Out of 22 acquired data sets in a given measurement session, five show an EMCD-like spectral signature. Although most of the other data sets lead to noisy structures of low amplitude, there are several cases strongly contrasting with the expected EMCD spectral shape, which have a similar amplitude as those five EMCD-like difference spectra. This naturally casts doubts about the validity of the APR-EMCD detection in this stage.

Further insight has been obtained by tensor decomposition techniques, specifically the canonical polyadic decomposition (CPD; see Methods section). CPD systematically reveals two components (see Fig. 3d) in every single acquired data set. One of them has a clear EMCD-like spectral signature while the other consists of two peaks located at edge positions, both having approximately the same height. Raw maps corresponding to the latter component show its localization on lattice planes, Fig. 3f, thus suggesting some sort of electron channelling effects. We suggest that this component should be explained by non-dipole transitions, because its presence requires transition matrix elements with a qualitatively different dependence on the momentum transfer vectors than the dominant dipole transitions. The EMCD-like component does not show any clear localization, its map seemingly being dominated by noise. However, subtraction of the second component as an additional pre-processing step in the data analysis described above increases the detection rate of EMCD-like spectra to over $50 \%$, while it removes false positives, that is, difference spectra with non-EMCD spectral signature with comparable amplitude, Fig. 3h. See Supplementary Fig. 2, for a similar analysis for another measurement session, leading to the same conclusions.

Quantitative analysis of the results via sum rules $^{5,6}$ is complicated due to the high noise level. Supplementary Fig. 3 summarizes the cumulative sums of the difference spectra ${ }^{13}$ shown in Fig. 3 and Supplementary Fig. 2. Except for the six noisiest curves showing an erratic behaviour in the pre- and post-edge region of their cumulative sums, preventing the application of sum rules, the orbital to spin moment ratios 5,6 $m_{L} / m_{S}$ range between -0.1 and 0.3 with a mean value of 0.057 and a standard error of 0.030 (s.d. 0.120). The median value for the reduced and the whole data set is 0.050 and 0.072 , respectively. Within an error margin given by the standard error, these values are in agreement with expected values for bulk iron $^{13}$, supporting the interpretation of the extracted spectral differences as EMCD. We note that even though a few data sets lead to expected $m_{L} / m_{S}$ ratios on their own, an overall higher quality of data is needed to systematically obtain reliable $m_{L} / m_{S}$ ratios from individual data sets.

\section{Discussion}

We have successfully demonstrated the detection of EMCD signal in our data sets, at the expense of enhanced data processing. 

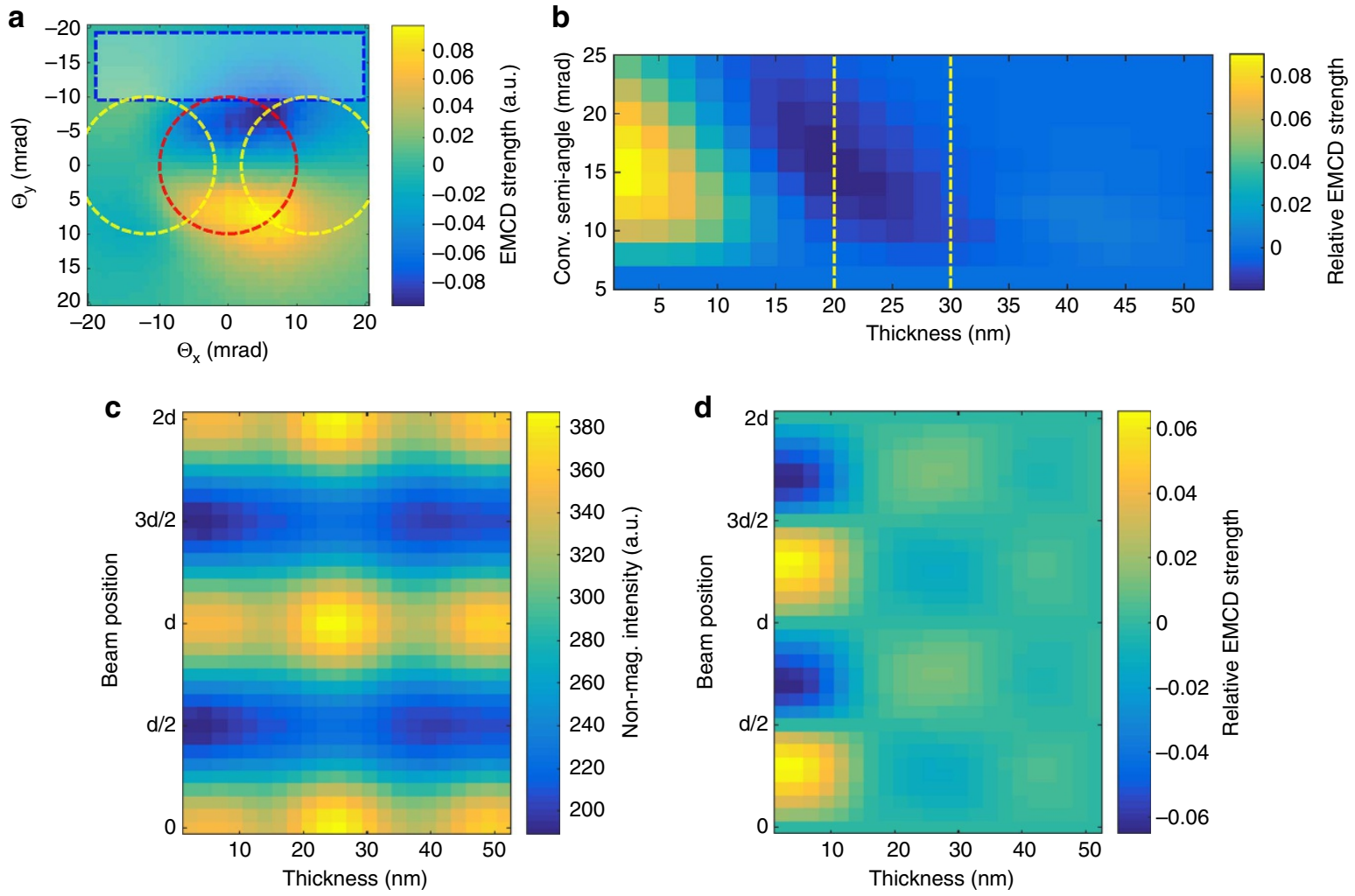

Figure 2 | Simulations of EMCD in the 3-beam geometry. (a) Example distribution of the Fe- $L_{3}$ edge EMCD signal in the diffraction plane for a beam positioned at $\frac{1}{4} d_{(110)}$ to the side from atomic planes. Positions of the transmitted beam disk (red circle) and Bragg scattered beam disks (yellow circles) are marked, as well as the rectangular detector region used in (b) the optimization of the relative EMCD signal strength as a function of convergence angle and sample thickness. (c) Calculated non-magnetic signal as a function of probe position and sample thickness at the Fe- $L_{3}$ edge and (d) the corresponding relative EMCD strength. In $\mathbf{b}, \mathbf{c}$ the convergence semi-angle was set to $10 \mathrm{mrad}$.

a

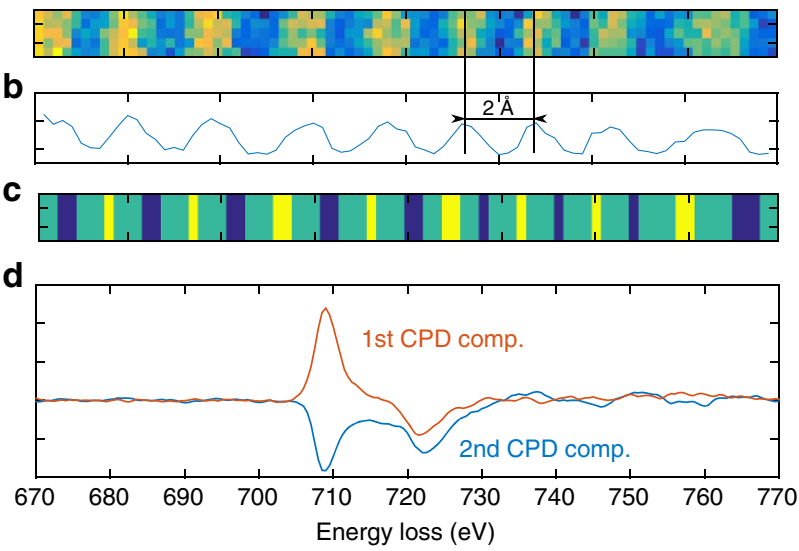

e
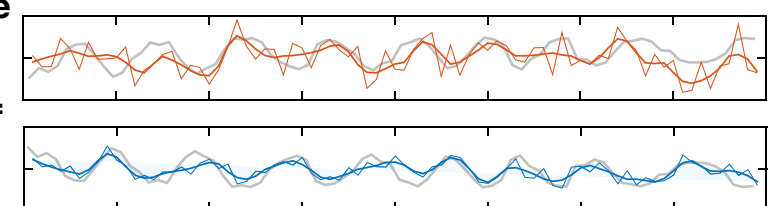

g

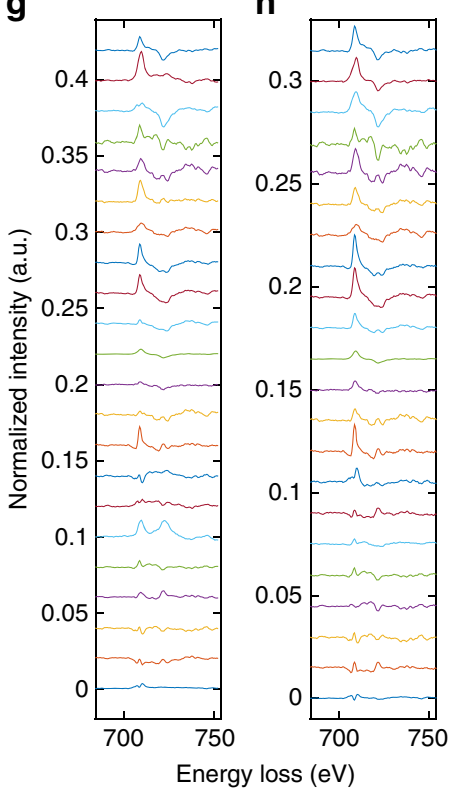

Figure 3 | Experimental detection of APR-EMCD. (a) Representative ADF image of the scanned area and (b) its line profile. A smoothed profile was used to identify individual minima and maxima, based on which a mask has been defined (c) with blue and yellow regions marking summation areas centred at $d / 4$ to the right or left of the atomic columns. Their width is determined by local steepness of the ADF line profile. (d) Example of the two CPD components with line profiles of their maps. (e,f) Note the strong correlation between the second CPD component (blue) and the overlayed ADF line profile shown in grey colour. The EMCD-like component also shows such a tendency, but the correlation is weaker. (g) Spectral differences obtained before the subtraction of the second CPD component and (h) after the subtraction. Note how the subtraction of the second CPD component leads to a higher number of spectra showing clear EMCD signature in (h). Spectral differences were calculated from scaled spectra, so that their average peak value at $L_{3}$ edge equals to one. $\ln \mathbf{g}$ and $\mathbf{h}$, the individual spectral differences were vertically offset by 0.02 and 0.015 , respectively. 
Nevertheless, the EMCD extraction could be greatly simplified by an efficient elimination of sample drift. Even small drifts can cause inclusion of spectra with negligible EMCD into the ROIs, thereby increasing the noise level and mixing in the channelling effects into spectral differences. We expect that these issues will be overcome by advanced drift correction algorithms and/or by rapid acquisitions of large data sets on instruments equipped with the needed functionality.

Importantly, our APR-EMCD measurement method does not require any modifications to the microscope hardware ${ }^{15}$ nor requires utilizing probe correctors in non-standard ways ${ }^{20}$, while it offers EMCD spectra at near-atomic resolution from an area as small as $0.5 \mathrm{~nm}^{2}$, a resolution that is likely to be even more enhanced in future experiments. We expect that its ease of implementation will greatly contribute to its proliferation and becoming the method of choice for magnetic measurements at the sub-nanometre scale.

\section{Methods}

Derivation of the EMCD strength in APR-EMCD set-up. For a thin sample, the inelastic scattering cross-section can be written as:

$$
\frac{\partial^{2} \sigma}{\partial E \partial \boldsymbol{\Omega}}=\sum_{\substack{\mathbf{k}, \mathbf{k}^{\prime}, \mathbf{G}, \mathbf{G}^{\prime} \\ k \perp, k^{\prime}<\alpha / \lambda}} T_{\mathbf{G}} T_{\mathbf{G}^{\prime}}^{*} e^{i\left(\phi_{\mathbf{k}}-\phi_{\mathbf{k}^{\prime}}\right)} S\left(\mathbf{q}, \mathbf{q}^{\prime}, E\right)
$$

where $\mathbf{q}=\mathbf{k}_{f}-\mathbf{k}-\mathbf{G}$ and $\mathbf{q}^{\prime}=\mathbf{k}_{f}-\mathbf{k}^{\prime}-\mathbf{G}^{\prime}$ and $S\left(\mathbf{q}, \mathbf{q}^{\prime}, E\right)$ is the mixed dynamical form factor ${ }^{22}$ (MDFF) describing intelastic transitions. For very thin samples $T_{\mathbf{G}}$ is $\sim 1$ for $\mathbf{G}=0$ and a small complex number for non-zero $\mathbf{G}$ vectors; $\phi_{\mathbf{k}}$ describes the phase distribution in the profile of the probe. Summing over atoms in a crystal leads to a condition that $\mathbf{q}-\mathbf{q}^{\prime}$ and consequently also $\mathbf{k}-\mathbf{k}^{\prime}$ needs to be a reciprocal lattice vector. Neglecting elastic scattering entirely we obtain

$$
\frac{\partial^{2} \sigma}{\partial E \partial \boldsymbol{\Omega}} \approx \sum_{\substack{\mathbf{k}, \mathbf{G} \\ k_{\perp}, k_{\perp}^{\prime}<\alpha / \lambda}} e^{i\left(\phi_{\mathbf{k}}-\phi_{\mathbf{k}+\mathbf{G}}\right)} S(\mathbf{q}, \mathbf{q}-\mathbf{G}, E),
$$

where $\mathbf{k}^{\prime}=\mathbf{k}+\mathbf{G}$. The magnetic signal is contained in the imaginary part of the MDFF (ref. 1) and the MDFF itself is Hermitian with respect to interchange of $\mathbf{q}$ and $\mathbf{q}^{\prime}$. Therefore we can write for the magnetic component of the scattering cross-section

$$
\left.\frac{\partial^{2} \sigma}{\partial E \partial \Omega}\right|_{\text {mag }} \approx \sum_{\substack{\mathbf{k}, \mathbf{G} \\ k_{\perp}, k_{\perp}^{\prime}<\alpha / \lambda}} \sin \left(\Delta \phi_{\mathbf{k}, \mathbf{G}}\right) \operatorname{Im}[S(\mathbf{q}, \mathbf{q}-\mathbf{G}, E)],
$$

where $\Delta \phi_{\mathbf{k}, \mathbf{G}}=\phi_{\mathbf{k}}-\phi_{\mathbf{k}+\mathbf{G}}$. In the 3-beam geometry we can orient the $x$-direction along the discrete periodicity-perpendicular to lattice planes-leading to $\mathbf{G}=(G, 0,0)$, which allows to express the imaginary part of the MDFF for magnetization along $z$-direction as

$$
\operatorname{Im}[S(\mathbf{q}, \mathbf{q}-\mathbf{G}, E)] \propto \frac{[\mathbf{q} \times(\mathbf{q}-\mathbf{G})]_{z} M}{q^{2} q^{\prime 2}}=\frac{-M G q_{y}}{q^{2} q^{\prime 2}}
$$

and the phase difference due to shifting the probe becomes $\Delta \phi_{\mathbf{k}, \mathbf{G}}=G \Delta x$. This shows that the EMCD signal oscillates as a function of displacement $\Delta x$ from the atomic planes with wavelength $d$, where $d$ is the distance between planes, and its distribution in the diffraction plane is antisymmetric with respect to the $x$ axis.

Dynamical diffraction simulations. A multislice method was used to describe elastic propagation of the convergent electron beam through an orthogonal supercell of bcc iron of dimensions $\sqrt{33} a \times \sqrt{2} a \times \sqrt{66} a$, so that the $c$ axis of this structure corresponds to propagation direction (11) and the $b$ axis is parallel to (110), perpendicular to the lattice planes. The spatial grid had a spacing of $0.068 \AA$ in all three dimensions, and we considered a three-dimensional potential parametrized by Weickenmeier and $\mathrm{Kohl}^{24}$. Inelastic calculations were performed using the modified automatic term selection (MATS) algorithm, using the multislice results to describe the elastically scattered incoming convergent probe ${ }^{23}$. The convergence parameter in the MATS summation algorithm was set to $10^{-6}$.

Sample preparation. 20 and 30 -nm-thick bcc iron layers covered by a 2 -nm-thick $\mathrm{Al}$ cap layer (to prevent the oxidation of $\mathrm{Fe}$ ) were deposited on 15-nm-thick $\mathrm{Si}_{3} \mathrm{~N}_{4}$ membranes by thermal evaporation in an ultra-high vacuum molecular beam epitaxy system. The thicknesses were controlled using calibrated quartz microbalances. No ex-situ or in-situ preparation/cleaning was applied to the $\mathrm{Si}_{3} \mathrm{~N}_{4}$ membranes before the deposition. The membranes were kept at room temperature during the deposition, but the Fe films were post-annealed at $750^{\circ} \mathrm{C}$ for $120 \mathrm{~min}$ before the deposition of the $\mathrm{Al}$ cap layers at room temperature. The disordered structure of the membranes (nanocrystalline or amorphous) results in a polycrystalline morphology of the metallic Fe/Al films. The post-annealing treatment led to a lateral grain size of the order $100 \mathrm{~nm}$. Air exposure between sample growth and EMCD measurements oxidized the Al cap layer to a depth of $\sim 1.5 \mathrm{~nm}$ ensuring a closed $\mathrm{AlO}_{x}$ layer. Accordingly, no substantial oxidation of the Fe was observable in both electron energy loss spectra (EELS) and electron diffraction. Some metallic Al may remain at the interface to the iron film.

Data acquisition. The measurements were carried out using EELS spectral imaging with an aberration-corrected scanning transmission electron microscope (STEM), the JEM ARM200F of the High-Voltage Electron Microscopy Laboratory, Nagoya University. The STEM was operated at $200 \mathrm{kV}$ and equipped with a Gatan Image Filter Quantum. The beam convergence semi-angle was $10 \mathrm{mrad}$ and the sample orientation was set to fulfil the symmetric 3-beam condition with $\mathbf{G}=(110)$, as shown in Fig. 1a, and a rectangular EELS entrance aperture was inserted as shown in Fig. 1d. The rectangular EELS aperture was implemented in the auxiliary slot next to the BF/ADF detectors of the Gatan Image Filter, which is conventionally used for a spatially-resolved EELS technique ${ }^{25}$. The spectrum image data sets were then obtained at a step width of $0.015 \mathrm{~nm}$ and an exposure of $0.2 \mathrm{~s}$ per spectrum, simultaneously collecting ADF images with the annular detector covering $50-90 \mathrm{mrad}$.

Data analysis. In the first step of the analysis, the data sets were aligned on their energy axis using a cross-correlation procedure. The noise influence was reduced using robust principal component analysis $\left(\mathrm{ROBPCA}^{26}\right)$ of the joint, aligned and mean-subtracted data set. Following the information of the scree plot, we reconstructed the data set using the first seven, most significant components. This estimate of the data dimension was also supported by the maximum likelihood estimator for the data dimension ${ }^{27}$. Subsequently, a power law background was subtracted from the data sets. Remaining sub-pixel instability of the $L_{2,3}$ peak positions were corrected for by orthogonalizing the data set to the derivative of the mean spectrum. Omitting this step leads to EMCD-like differences in some cases, however, the derivative of the mean spectrum often dominates the difference spectrum. Such pre-processed data sets were used as an input for the analysis via spectral difference described in the next paragraph. For the CPD (see below), the mean spectrum has been subtracted after prior post-edge normalization.

ROIs were identified on the basis of ADF images, see Fig. 3b,c. A line profile of ADF image was smoothed for easier identification of the position of atomic planes. The local maxima of the line profile represent positions of atomic planes and local minima represent the region in the middle between two neighbouring planes. Then the ROIs have their centres in the midpoints between the neighbouring pairs of minima and maxima. The width of each ROI was chosen as $40 \%$ of the distance between its enclosing minimum and maximum. An example map of the ROIs is shown in Fig. 3c. Thus defined ROIs fall into two categories, being right or left from their closest atomic planes. Theory predicts opposite signs of EMCD contained in these two categories of ROIs. Consequently, two spectra are formed as averages of all individual spectra falling into these two groups of ROIs. Those two spectra are post-edge normalized and finally their difference is extracted, as shown in Fig. 3g.

Analysis via CPD (ref. 28) is performed as the last step of the data processing of the individual spectrum images, before extraction of the spectra using ROIs described in the previous paragraph. Its sole purpose is removal of the component shown in Fig. 3d in blue colour from the spectrum images. CPD provides a unique decomposition (in the mathematical sense), which is a desirable property. In CPD, each data point of a three-dimensional datacube, $p_{k, l, m}$, is described as

$p_{k, l, m}=\sum_{i} a_{k}^{(i)} b_{l}^{(i)} c_{m}^{(i)}$ for a given number of components in a way that minimizes the sum of the squares of the residuals. Each of the terms in the sum is a datacube, where the information along each mode can be described by a single vector. Here, $\mathrm{CPD}$ is invoked for matricized datacubes and the third dimension is represented by the original data set with a small level of noise modelled as a uniformly distributed random variable. Before being subjected to the $\mathrm{CPD}$, the resulting tensor was compressed using multilinear singular value decomposition. Due to the prior truncation via ROBPCA and further pretreatment, six components along spatial and energy modes suffice to capture the contained information. Two-component CPD leads reproducibly for all measured datacubes to the same two spectral components as shown in Fig. 3d.

Data availability. All relevant data are available from authors.

\section{References}

1. Schattschneider, P. et al. Detection of magnetic circular dichroism using a transmission electron microscope. Nature 441, 486-488 (2006).

2. Hébert, C. \& Schattschneider, P. A proposal for dichroic experiments in the electron microscope. Ultramicroscopy 96, 463-468 (2003).

3. Schütz, G. et al. Absorption of circularly polarized $\mathrm{x}$ rays in iron. Phys. Rev. Lett. 58, 737-740 (1987). 
4. Rusz, J., Rubino, S. \& Schattschneider, P. First-principles theory of chiral dichroism in electron microscopy applied to $3 \mathrm{~d}$ ferromagnets. Phys. Rev. B 75, 214425 (2007).

5. Rusz, J., Eriksson, O., Novák, P. \& Oppeneer, P. M. Sum rules for electron energy loss near edge spectra. Phys. Rev. B 76, 060408 (R) (2007).

6. Calmels, L. et al. Experimental application of sum rules for electron energy loss magnetic chiral dichroism. Phys. Rev. B 76, 060409 (R) (2007).

7. Schattschneider, P. et al. Detection of magnetic circular dichroism on the two-nanometer scale. Phys. Rev. B 78, 104413 (2008).

8. Salafranca, J. et al. Surfactant organic molecules restore magnetism in metal-oxide nanoparticle surfaces. Nano Lett. 12, 2499-2503 (2012).

9. Schattschneider, P. et al. Magnetic circular dichroism in EELS: Towards $10 \mathrm{~nm}$ resolution. Ultramicroscopy 108, 433-438 (2008).

10. Warot-Fonrose, B. et al. Mapping inelastic intensities in diffraction patterns of magnetic samples using the energy spectrum imaging technique. Ultramicroscopy 108, 393-398 (2008).

11. Lidbaum, H. et al. Quantitative magnetic information from reciprocal space maps in transmission electron microscopy. Phys. Rev. Lett. 102, 037201 (2009).

12. Wang, Z. Q., Zhong, X. Y., Yu, R., Cheng, Z. Y. \& Zhu, J. Quantitative experimental determination of site-specific magnetic structures by transmitted electrons. Nat. Commun. 4, 1395 (2013).

13. Muto, S. et al. Quantitative characterization of nanoscale polycrystalline magnets with electron magnetic circular dichroism. Nat. Commun. 5, 3138 (2014).

14. Thersleff, T. et al. Quantitative analysis of magnetic spin and orbital moments from an oxidized iron $\left(\begin{array}{lll}1 & 1 & 0\end{array}\right)$ surface using electron magnetic circular dichroism. Sci. Rep. 5, 13012 (2015).

15. Verbeeck, J., Tian, H. \& Schattschneider, P. Production and application of electron vortex beams. Nature 467, 301-304 (2010).

16. Uchida, M. \& Tonomura, A. Generation of electron beams carrying orbital angular momentum. Nature 464, 737-739 (2010).

17. McMorran, B. J. et al. Electron vortex beams with high quanta of orbital angular momentum. Science 331, 192-195 (2011).

18. Rusz, J. \& Bhowmick, S. Boundaries for efficient use of electron vortex beams to measure magnetic properties. Phys. Rev. Lett. 111, 105504 (2013).

19. Schattschneider, P., Löffler, S., Stöger-Pollach, M. \& Verbeeck, J. Is magnetic chiral dichroism feasible with electron vortices? Ultramicroscopy 136, 81-85 (2014).

20. Rusz, J., Idrobo, J. C. \& Bhowmick, S. Achieving atomic resolution magnetic dichroism by controlling the phase symmetry of an electron probe. Phys. Rev. Lett. 113, 145501 (2014).

21. Idrobo, J. C. et al. Detecting magnetic ordering with atomic size electron probes. Adv. Struct. Chem. Imag. 2, 5 (2016).

22. Kohl, H. \& Rose, H. Theory of image formation by inelastically scattered electrons in the electron microscope. Adv. Electron. Electron Opt. 65, 173-226 (1985).

23. Rusz, J., Bhowmick, S., Eriksson, M. \& Karlsson, N. Scattering of electron vortex beams on a magnetic crystal: Towards atomic-resolution magnetic measurements. Phys. Rev. B 89, 134428 (2014).

24. Weickenmeier, A. \& Kohl, H. Computation of absorptive form factors for high-energy electron diffraction. Acta Cryst. A47, 590-597 (1991).

25. Kimoto, K., Sekiguchi, T. \& Aoyama, T. Chemical shift mapping of Si L and K edges using spatially resolved EELS and energy filtering TEM. J. Electron Microsc. 46, 369-374 (1997).
26. Hubert, M., Rousseeuw, P. J. \& Vanden Branden, K. ROBPCA: a new approach to robust principal component analysis. Technometrics 47, 64-79 (2005).

27. Levina, E. \& Bickel, P. J. in Advances in Neural Information Processing Systems Vol. 17, 777-784 (The MIT Press, 2004).

28. Cichocki, A. et al. Tensor decompositions for signal processing applications: from two-way to multiway component analysis. IEEE Signal Process. Mag. 32, 145-163 (2015).

\section{Acknowledgements}

This work was in part supported by Grant-in-Aid for Scientific Research of JSPS, the Swedish Research Council, the Göran Gustafsson's foundation, the K. and A. Wallenberg Foundation (project no. 2015.0060) and Japan-Sweden Joint Research Program by JSPS and STINT. A part of this work was supported by Nagoya University microstructural characterization platform as a program of Nanotechnology Platform of the Ministry of Education, Culture, Sports, Science and Technology (MEXT), Japan. J.S. acknowledges the Center of Interdisciplinary Mathematics. J.R. acknowledges A. Magnard and B. Violinist for helpful discussions on correlated noise.

\section{Author contributions}

J.R., S.M. and K.T. discussed utility of large convergence angles for EMCD measurements in 3-beam orientation, leading to simulations and discovery of the beam shift method. J.R. provided theoretical background and simulations, S.M. measured the spectrum images, J.S. developed software routines for data analysis and analysed the data, K.T. constructed the supercell structure model and contributed to simulations. P.M.O. initiated and coordinated the cooperation between Nagoya and Jülich research groups. R.A., D.E.B. and C.M.S. prepared the samples. All authors discussed the results and contributed to writing the manuscript.

\section{Additional information}

Supplementary Information accompanies this paper at http://www.nature.com/ naturecommunications

Competing financial interests: The authors declare no competing financial interests.

Reprints and permission information is available online at http://npg.nature.com/ reprintsandpermissions/

How to cite this article: Rusz, J. et al. Magnetic measurements with atomic-plane resolution. Nat. Commun. 7:12672 doi: 10.1038/ncomms12672 (2016).

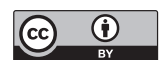

This work is licensed under a Creative Commons Attribution 4.0 International License. The images or other third party material in this article are included in the article's Creative Commons license, unless indicated otherwise in the credit line; if the material is not included under the Creative Commons license, users will need to obtain permission from the license holder to reproduce the material. To view a copy of this license, visit http://creativecommons.org/licenses/by/4.0/

(C) The Author(s) 2016 


\section{Erratum: Magnetic measurements with atomic-plane resolution}

Ján Rusz, Shunsuke Muto, Jakob Spiegelberg, Roman Adam, Kazuyoshi Tatsumi, Daniel E. Bürgler,

Peter M. Oppeneer \& Claus M. Schneider

Nature Communications 7:12672 doi: 10.1038/ncomms12672 (2016); Published 31 Aug 2016; Updated 5 Oct 2016

In the originally published version of the Article, an incorrect reference number was cited. The final sentence of the first paragraph of the Introduction section should read: 'The likely reasons are technical difficulties in generating atomic size electron vortex beams, together with fine control of their resulting orbital angular momentum in the case of electron vortex beams, and a low EMCD signal fraction in the case of astigmatic beams ${ }^{21}$.

(c) (i) This work is licensed under a Creative Commons Attribution 4.0 International License. The images or other third party material in this article are included in the article's Creative Commons license, unless indicated otherwise in the credit line; if the material is not included under the Creative Commons license, users will need to obtain permission from the license holder to reproduce the material. To view a copy of this license, visit http://creativecommons.org/licenses/by/4.0/

(C) The Author(s) 2016 